\title{
Characterization of the clonal profile of MRSA isolated in neonatal and pediatric intensive care units of a University Hospital
}

\author{
Valéria Cataneli Pereira*, Danilo Flávio Moraes Riboli and Maria de Lourdes Ribeiro de Souza da Cunha
}

\begin{abstract}
Background: Methicillin-resistant Staphylococcus aureus (MRSA) are important pathogens in neonatal and pediatric intensive care units, which can cause severe infections in hospitalized children. Detection of the mecA gene and classification of the staphylococcal cassette chromosome mec (SCCmec) permit the characterization of MRSA strains isolated from infections caused by these microorganisms. In contrast, pulsed-field gel electrophoresis (PFGE) is used to type MRSA clones. This method is commonly used to analyze the epidemiology of bacteria causing nosocomial infections. The objective of this study was to detect and characterize MRSA isolated from clinical specimens of children hospitalized in the neonatal and pediatric intensive care units of the University Hospital of the Botucatu Medical School.
\end{abstract}

Methods: A total of 119S. aureus strains were isolated from clinical specimens and the mecA gene was detected by PCR. SCCmec was detected by multiplex PCR and the clonal profile was analyzed by PFGE.

Results: The mecA gene was detected in 17.6\% (21/119) of the isolates; $42.9 \%$ (9/21) of MRSA were characterized as SCCmec type III and 57.1\% (12/21) as type IV. Analysis of the clonal profile of these strains revealed three distinct clones, with SCCmec type III being related to the Brazilian endemic clone and type IV to clones JCSC4469 and USA800.

Conclusions: Replacement of clonal groups occurred in the neonatal and pediatric units over the period studied, a fact highlighting the importance of improving hygiene practices and control measures of nosocomial infections in these units.

Keywords: Nosocomial infections, NICU, PICU, MRSA

\section{Background}

The genus Staphylococcus is a member of the family Staphylococcaceae, which comprises 49 species and 26 subspecies [1,2]. Staphylococcus aureus is the most important species of this genus and the causative agent of a range of infections, such as furuncles, cellulitis, impetigo, and wound infections. Some of the most severe infections caused by $S$. aureus include bacteremia, pneumonia, osteomyelitis, acute endocarditis, myocarditis, meningitis, and abscesses in muscles, genitourinary tract, central nervous system and various intra-abdominal organs $[3,4]$.

\footnotetext{
* Correspondence: cataneli@ibb.unesp.br

Laboratory of Bacteriology, Department of Microbiology and Immunology, Institute of Biosciences, UNESP - Univ Estadual Paulista, Botucatu, São Paulo CEP 18618-970, Brazil
}

Studies have shown that 60 to $85 \%$ of staphylococci isolated from clinical samples are resistant to methicillin [5]. Methicillin-resistant Staphylococcus aureus strains (MRSA) are important pathogens in neonatal (NICU) and pediatric intensive care units (PICU), which can cause severe infections in hospitalized children who are generally exposed to several risk factors, such as prematurity, invasive procedures, mechanical ventilation, and drains [6].

Oxacillin is the drug of choice for susceptibility testing and treatment of infections caused by Staphylococcus. Intrinsic resistance of $S$. aureus to oxacillin is mediated by the production of a supplemental penicillin-binding protein (PBP 2a), which is encoded by the mecA gene [7]. This gene is found on a specific mobile genetic element 
identified as the staphylococcal cassette chromosome mec (SCCmec), which consists of the mecA gene complex, $c c r$ gene complex, and region J. The mec complex comprises the mecA gene and its regulatory genes mecI and mecRI. The $c c r$ gene complex is responsible for the integration and excision of SCCmec in the chromosome. In contrast, region $\mathrm{J}$ is not essential for the cassette chromosome, but can carry genes that encode resistance to non-beta-lactam antibiotics and heavy metals [8]. Eleven SCCmec types have been described so far [9]. These types are defined based on the combination of the type of $c \mathrm{cr}$ gene complex and class of the mec gene complex. Subtypes are defined based on polymorphisms in region J of the same combination of mec and ccr complexes [8].

SCCmec types I, II and III are classically found in nosocomial MRSA strains, whereas the other types are found in community-associated MRSA [10]. SCCmec type III encodes the largest number of resistance genes and strains harboring this type are important pathogens in hospitals where they cause severe infections [11]. In contrast, type IV is characterized by a smaller size and lower metabolic cost, a fact selectively favoring this element for transfer between staphylococci [12]. Communityassociated MRSA have been reported to cause severe infections in NICU and PICU patients who never have been hospitalized [6]. According to these authors, the most frequent complications caused by these microorganisms are pneumonia and skin and soft tissue infections and strains carrying SCCmec type IV are the most common [6].

The increasing occurrence of MRSA in hospitals makes the typing of these microorganisms important in order to determine whether the strains involved in nosocomial infections or in possible foci of transmission are related to a specific clone, i.e., whether they have a common origin [13]. Pulsed-field gel electrophoresis (PFGE) is commonly used to analyze the epidemiology of bacteria causing nosocomial infections. This method permits to clearly discriminate strains and to demonstrate the genetic relationship between isolates with high reproducibility [13]. The objective of the present study was to detect and characterize MRSA isolated from clinical specimens of children hospitalized in the NICU and PICU of the University Hospital of the Botucatu Medical School (HC-FMB).

\section{Methods}

\section{Strains}

A total of $119 \mathrm{~S}$. aureus strains isolated from clinical specimens of children hospitalized in the NICU and PICU of HC-FMB between 1991 and 2009 were studied. Thirty-nine of the 76 neonatal strains were isolated from blood cultures, 22 from secretions, 12 from catheters, and three from cannulae. In the pediatric ward, 41 of the
43 strains were isolated from blood cultures, one from pleural fluid, and one from peritoneal fluid. The strains were isolated as described by Koneman et al. [14] on blood agar plates (Blood Agar Base, Himedia, Mumbai, India) and suspected colonies were submitted to Gram staining. After confirmation of morphology and specific staining, the isolates were identified using catalase and coagulase tests.

\section{DNA extraction}

Total nucleic acid was extracted from $S$. aureus isolates cultured on blood agar (Blood Agar Base, Himedia, Mumbai, India), inoculated individually into brain-heart infusion broth (Oxoid Ltd., Basingstoke, Hampshire, England), and incubated for $24 \mathrm{~h}$ at $37^{\circ} \mathrm{C}$. Extraction was performed using the Illustra kit (Illustra ${ }^{\mathrm{Tm}}$, GE Healthcare, Pittsburg, PA, USA), which consisted of initial digestion of bacterial cells with lysozyme (Amresco ${ }^{\circ}$, Solon, Ohio, USA) $(10 \mathrm{mg} / \mathrm{mL})$ and proteinase $\mathrm{K}$ (GE Healthcare, Pittsburg, PA, USA) $(20 \mathrm{mg} / \mathrm{mL})$. Five hundred $\mu \mathrm{L}$ of the extraction solution (Illustra $^{\text {Tx }}$, GE Healthcare, Pittsburg, PA, USA) was added and the mixture was centrifuged (Centrifuge $5804 \mathrm{R}$, Eppendorf AG, Hamburg, Germany) at 5,000 $\times g$ for $1 \mathrm{~min}$. The supernatant was transferred to a column and centrifuged (Centrifuge 5804 R, Eppendorf AG, Hamburg, Germany) at 5,000 $\times g$ for $1 \mathrm{~min}$. The collected fluid was discarded and $500 \mu \mathrm{L}$ extraction solution (Illustra ${ }^{\mathrm{Tm}}$, GE Healthcare, Pittsburg, PA, USA) was added again to the column. After centrifugation and discarding of the collected fluid, $500 \mu \mathrm{L}$ washing solution (Illustra ${ }^{\mathrm{Tu}}$, GE Healthcare, Pittsburg, PA, USA) was added and the column was centrifuged (Centrifuge 5804 R, Eppendorf AG, Hamburg, Germany) at $20,817 \times g$ for $3 \mathrm{~min}$. The column was transferred to a $1.5-\mathrm{mL}$ tube and $200 \mu \mathrm{L}$ Milli-Q water heated to $70^{\circ} \mathrm{C}$ was used for elution. The samples were centrifuged (Centrifuge 5804 R, Eppendorf AG, Hamburg, Germany) at $5,000 \times g$ for $1 \mathrm{~min}$ and the column was discarded. The extracted DNA was stored in a refrigerator (Brastemp BRD45, Whirlpool S.A., São Paulo, Brazil) at $4^{\circ} \mathrm{C}$.

\section{Detection of the mecA gene}

The $m e c$ A gene was investigated in the S. aureus isolates for detection of oxacillin resistance. The primers and parameters described by Murakami et al. [15] were used for amplification: primers mecA1 (AAA ATC GAT GGT AAA GGT TGG) and mecA2 (AGT TCT GCA GTA CCG GAT TTG) that amplify a fragment of $533 \mathrm{bp}$. International reference strains were included as positive (S. aureus ATCC 33591) and negative (S. aureus ATCC 25923) controls in all reactions.

\section{Determination of the SCCmec type}

The SCCmec type was determined in the MRSA isolates by multiplex PCR. The primers and parameters 
described by Milheiriço et al. [16] were used for amplification.

\section{Pulsed-field gel electrophoresis}

The clonal profile of the Staphylococcus spp. isolates was determined using the modified protocol of McDougal et al. [17]. The strains were inoculated into brain-heart infusion broth (Oxoid Ltd., Basingstoke, Hampshire, England) and incubated for $24 \mathrm{~h}$ at $37^{\circ} \mathrm{C}$. The isolates were centrifuged (Centrifuge 5804 R, Eppendorf AG, Hamburg) in microtubes at $15,294 \times g$ for $1 \mathrm{~min}$, the supernatant was discarded, $300 \mu \mathrm{L}$ TE solution (10 mM Tris, $1 \mathrm{mM}$ EDTA, pH 8.0) was added, and the strains were kept in a water bath for $10 \mathrm{~min}$ at $37^{\circ} \mathrm{C}$. The cells were lysed by the addition of $5 \mu \mathrm{L}$ lysostaphin (from Staphylococcus lyophilized powder, Sigma-Aldrich) and vortexed (Phoenix AP-56), and $300 \mu \mathrm{L}$ of $1.8 \%$ low-melt agarose (Agarose-Low Melt, USB Corporation, Ohio, USA) was added at $37^{\circ} \mathrm{C}$. Plugs were prepared from the strains and the agarose (Agarose-Low Melt, USB Corporation, Ohio, USA) was allowed to solidify. The plugs were transferred to a 24well plate containing $2 \mathrm{~mL}$ EC solution $(6 \mathrm{mM}$ Tris- $\mathrm{HCl}$, $1 \mathrm{M} \mathrm{NaCl}, 100 \mathrm{mM}$ EDTA, 0.5\% Brij-58, 0.2\% sodium deoxycholate, $0.5 \%$ sodium lauroyl sarcosinate) and incubated for $4 \mathrm{~h}$ at $37^{\circ} \mathrm{C}$. The EC solution $(6 \mathrm{mM}$ Tris- $\mathrm{HCl}$, $1 \mathrm{M} \mathrm{NaCl}, 100 \mathrm{mM}$ EDTA, 0.5\% Brij-58, 0.2\% sodium deoxycholate, $0.5 \%$ sodium lauroyl sarcosinate) was removed and the plugs were washed four times in $2 \mathrm{~mL}$ TE solution (10 mM Tris, $1 \mathrm{mM}$ EDTA, $\mathrm{pH}$ 8.0) for $30 \mathrm{~min}$ at $21^{\circ} \mathrm{C}$.

One-third of the plug and $2 \mu \mathrm{L}$ SmaI (Fast Digest SmaI, Thermo Scientific, Lithuania, EU) were used for the restriction of genomic DNA. For restriction, buffer without the enzyme ( $45 \mu \mathrm{L}$ Milli-Q water and $5 \mu \mathrm{L}$ of the enzyme buffer) was added to a 96-well plate and the plate was stored in a refrigerator (Brastemp BRD45, Whirlpool S.A., São Paulo, Brazil) for $30 \mathrm{~min}$ at $4^{\circ} \mathrm{C}$. The buffer without enzyme was removed and buffer containing the enzyme ( $43 \mu \mathrm{L}$ Milli-Q water, $5 \mu \mathrm{L}$ enzyme buffer, and $2 \mu \mathrm{L}$ of the enzyme) was added. The plate was incubated in an oven (Eletrolab $101 \mathrm{M} / 3$, São Paulo, Brazil) for $6 \mathrm{~min}$ at $37^{\circ} \mathrm{C}$. Electrophoresis was carried out in a CHEF-DR III System (BioRad Laboratories, Hercules, California USA) using 1\% agarose gel (PulsedField Certified Agarose, BioRad Laboratories, USA) prepared in $0.5 \mathrm{M}$ TBE $(0.1 \mathrm{M}$ Tris, $0.08 \mathrm{M}$ boric acid, $1 \mathrm{mM}$ EDTA) under the following conditions: pulse times of 5 to $40 \mathrm{~s}$ for $21 \mathrm{~h}$ on a linear ramp; $6 \mathrm{~V} / \mathrm{cm}$; angle of $120^{\circ} ; 14^{\circ} \mathrm{C} ; 0.5 \mathrm{M} \mathrm{TBE}$ as running buffer. The Lambda Ladder PFG Marker (New England BioLabs, Hitchin, United Kingdom) was used as a molecular marker. The gels were stained with GelRed $(400 \mathrm{~mL}$ distilled, water and $30 \mu \mathrm{L}$ GelRed) $(10,000 \mathrm{X}$ in water,
Biotium, Hayward, CA) for $1 \mathrm{~h}$ and photographed under UV transillumination.

The BioNumerics software, version 6.1 (Applied Maths, Belgium), was used for analysis of similarity, calculation of the Dice correlation coefficient, and construction of the dendrogram by the UPGMA method (unweighted pair group method using arithmetic averages). Band position tolerance and optimization were set at 1.25 and $0.5 \%$, respectively. A similarity coefficient of $80 \%$ was chosen for the definition of clusters.

International clones kindly provided by Dr. Antonio Carlos Campos Pignatari, Laboratório Especial de Microbiologia Clínica, Disciplina de Infectologia, Universidade Federal de São Paulo/Escola Paulista de Medicina, and by Dr. Agnes Marie Sá Figueiredo, Universidade Federal do Rio de Janeiro, Instituto de Microbiologia Prof. Paulo de Góes, Brazil, were used as controls: USA800 (SCCmec IVa), JCSC 1968/CA05 (SCCmec IVa), JCSC 978/8/6-3P (SCCmec IVb), MR108 (SCCmec IVc), JCSC 4469 (SCCmec IVd), WB72/USA300 (SCCmec IV), USA400 (SCCmec IV), USA500 (SCCmec IV), OSPC (SCCmec IV), HAR24/ EMRSA 15 (SCCmec IV), HU25 (SCCmec IIIa), 85/2082 (SCCmec III), and ANS 46 (SCCmec III).

\section{Results}

The mecA gene was detected in $17.6 \%(21 / 119)$ of the $S$. aureus isolates studied. MRSA were detected in $18.4 \%$ $(14 / 76)$ of the S. aureus strains isolated from the NICU, including seven strains isolated from blood cultures, four from secretions, and three from catheters. Seven 16.3\% (7/43) strains from the PICU carried the mecA gene, including six strains isolated from blood cultures and one strain isolated from pleural fluid (Table 1).

\section{Characterization of the staphylococcal cassette chromosome mec}

The 21 mecA gene-positive $S$. aureus isolates were submitted to multiplex PCR for characterization of the SCCmec type. Nine of the 21 strains (42.9\%) were classified as type III and $12(57.1 \%)$ as type IV. Eight of the nine MRSA type III strains were isolated from clinical specimens of children hospitalized in the NICU and one in the PICU. Six of the type IV strains were isolated in the NICU and six in the PICU (Table 1).

\section{Evolution of oxacillin resistance in S. aureus strains isolated from patients seen at HC-FMB}

Analysis of the period from 1991 to 2009 showed the early presence of SCCmec type IV in a strain isolated in 1993. Although the sample size of this study was too small to detect a significant difference, the results showed a decrease in the prevalence of SCCmec type III and a recent increase in SCCmec type IV-carrying isolates. 
Table 1 Detection of MRSA and SCCmec type according to hospital ward and clinical specimens

\begin{tabular}{|c|c|c|c|c|c|c|c|c|}
\hline & \multicolumn{4}{|c|}{ Neonatal intensive care unit } & \multicolumn{4}{|c|}{ Pediatric intensive care unit } \\
\hline & \multirow[t]{2}{*}{$\mathrm{N}$} & \multirow[t]{2}{*}{$\%$ MRSA } & \multicolumn{2}{|c|}{ SCCmec type } & \multirow[t]{2}{*}{$\mathrm{N}$} & \multirow[t]{2}{*}{$\%$ MRSA } & \multicolumn{2}{|c|}{ SCCmec type } \\
\hline & & & Type III & Type IV & & & Type III & Type IV \\
\hline Blood culture $(\mathrm{N}=80)$ & 39 & 17.5 & 2 & 5 & 41 & 14.6 & 1 & 5 \\
\hline Secretion $(N=22)$ & 22 & 18.2 & 3 & 1 & 0 & 0 & 0 & 0 \\
\hline Fluid $^{\mathbf{a}}(\mathrm{N}=2)$ & 0 & 0 & 0 & 0 & 2 & 50.0 & 0 & 1 \\
\hline Foreign body ${ }^{\mathbf{b}}(\mathrm{N}=15)$ & 15 & 20.0 & 3 & 0 & 0 & 0 & 0 & 0 \\
\hline
\end{tabular}

$\mathrm{N}$ : number of strains.

${ }^{\mathrm{a}}$ Peritoneal and pleural fluid.

${ }^{\mathrm{b}}$ catheter and cannula.

Analysis of the clonal profile of MRSA

Analysis of the clonal profile of the MRSA strains isolated in this study revealed four distinct clones. MRSA harboring SCCmec type III were divided into two groups, one related to the Brazilian endemic clone (HU25). The strains carrying SCCmec type IV were also divided into two groups, one related to a clone found in the United States (USA800) and the other related to a clone found in Japan (JCSC4469) (Figure 1).

\section{Discussion}

Oxacillin-resistant Staphylococcus aureus are important pathogens involved in infections that affect children hospitalized in intensive care units in many countries. Although the frequency of oxacillin resistance is high among S. aureus strains, particularly in large hospitals and universities, the frequency of isolation of MRSA in the NICU and PICU of HC-FMB was 17.6\% (21/119) over a period of 18 years; $18.4 \%$ (14/76) of these isolates were

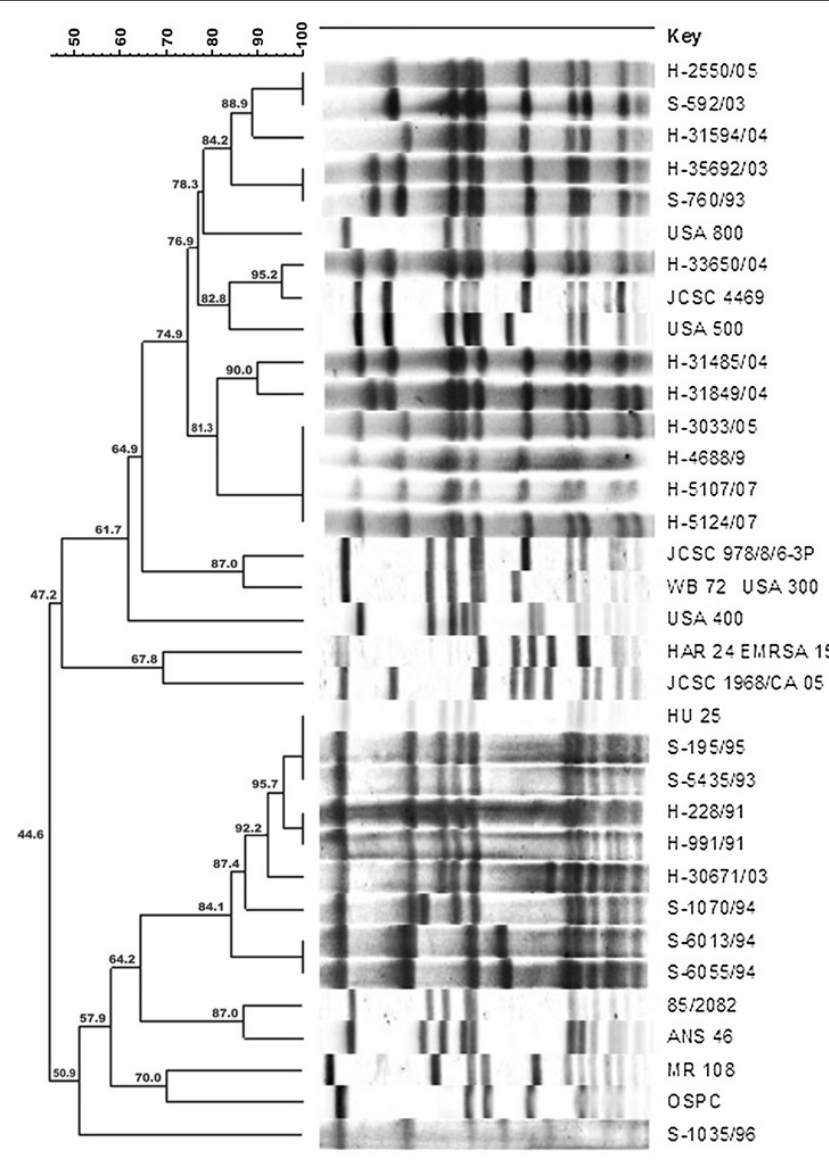

\begin{tabular}{|c|c|c|}
\hline Material & scCmec & Procedence \\
\hline Blood culture & IV & PICU \\
\hline Secretion & IV & PICU \\
\hline Blood culture & IV & PICU \\
\hline Blood culture & IV & PICU \\
\hline \multirow[t]{2}{*}{ Secretion } & IV & NICU \\
\hline & IVa & \\
\hline \multirow[t]{3}{*}{ Blood culture } & IV & NICU \\
\hline & IVd & \\
\hline & IV & \\
\hline Blood culture & IV & PICU \\
\hline Blood culture & IV & PICU \\
\hline Blood culture & IV & NICU \\
\hline Blood culture & IV & NICU \\
\hline Blood culture & IV & NICU \\
\hline \multirow[t]{7}{*}{ Blood culture } & IV & NICU \\
\hline & IVo & \\
\hline & IV & \\
\hline & IV & \\
\hline & IV & \\
\hline & IVa & \\
\hline & IIIa & \\
\hline Secretion & III & NICU \\
\hline Secretion & III & NICU \\
\hline Blood culture & III & NICU \\
\hline Blood culture & III & NICU \\
\hline Blood culture & III & PICU \\
\hline Secretion & III & NICU \\
\hline Secretion & III & $\mathrm{NICU}$ \\
\hline \multirow[t]{5}{*}{ Secretion } & III & NICU \\
\hline & III & \\
\hline & III & \\
\hline & IVc & \\
\hline & IV & \\
\hline Secretion & III & $\mathrm{NICU}$ \\
\hline
\end{tabular}

Figure 1 Determination of the clonal profile of MRSA carrying SCCmec type III and type IV isolated from clinical specimens of children hospitalized in the neonatal (NICU) and pediatric (PICU) intensive care units of HC-FMB. 
detected in the NICU and 16.3\% (7/43) in the PICU. Similar results have been reported in a study conducted in the United Kingdom, in which S. aureus strains isolated in the NICU and PICU of a hospital over a period of 10 years (1993 to 2003) were analyzed. The frequency of isolation of MRSA related to bacteremia was $15.1 \%$ (5/33) [18]. In a study conducted in New Zealand, the frequency of isolation of MRSA was $12.0 \%(7 / 58)$ in a PICU over a period of 11 years (1993 to 2004) [19]. In contrast, different results were found in the NICU of a hospital in the United States where $47.4 \%$ (8/17) of MRSA were detected among S. aureus over a period of 10 years (2000 to 2009) [20].

In the two wards, the $S$. aureus strains isolated from blood cultures exhibited a similar percentage of oxacillin resistance [NICU: 17.5\% (7/39), PICU: 14.6\% (6/41)]. In the NICU, MRSA were also isolated from other clinical specimens such as secretions $[18.2 \%(4 / 22)]$ and catheters and cannula $[20.0 \%(3 / 15)]$. In the PICU, only one strain isolated from pleural fluid was resistant to oxacillin.

Among the MRSA detected in this study, 57.1\% (12/21) were characterized as SCCmec type IV; of these, 83.3\% $(10 / 12)$ were isolated from blood cultures. In the study of Healy et al. [21], 75\% (6/8) of MRSA strains isolated in the NICU were typed as SCCmec type IV. SCCmec type IV is the most frequent type found in the community and is also becoming predominant among healthcareassociated MRSA infections $[8,22,23]$. The smaller size of the cassette chromosome when compared to types I, II and III probably increases its mobility and transfer capacity between Staphylococcus, suggesting that clones carrying this SCCmec element may spread more easily and that diseases caused by these strains tend to increase $[24,25]$. According to Dolapo et al. [20], the incidence of MRSA infections in NICUs is still unacceptably high. This fact may be related to the acquisition of communityassociated MRSA strains, which have evolved in the community and penetrated the NICU through parents or care providers.

SCCmec type III was identified in $42.9 \%(9 / 21)$ of MRSA and predominated among strains isolated in the 1990s. Only one strain was detected after 2000. SCCmec type III is commonly found in Brazilian hospitals and is highly resistant to various antimicrobial agents used to treated $S$. aureus infections, including resistance to beta-lactams, macrolides, aminoglycosides and trimethoprim-sulfamethoxazole [26]. In the study of Perez \& D'Azevedo [27], nine MRSA were susceptible only to vancomycin, linezolid and teicoplanin. Eight of these strains carried SCCmec type III.

In the present study, SCCmec typing permitted to confirm the isolation of two types of MRSA in the NICU and PICU of HC-FMB over a period of 18 years. One important finding was the isolation of MRSA carrying SCCmec type IV in 1993 from the secretion sample of a newborn. SCCmec type IV was only typed again 10 years later in the pleural fluid sample of a child hospitalized in the pediatric unit. From that time on, this SCCmec was the predominant type among all MRSA isolated in the two units. According to Milheiriço et al. [16], the SCCmec element is an important marker for the determination of MRSA clones. In addition to being a valuable tool for the study of MRSA epidemiology, SCCmec characterization permits to investigate the evolution of MRSA clones in culture collections.

With respect to the epidemiology and evolution of MRSA clones, PFGE permitted a better analysis of the data obtained in this study. The MRSA isolates carrying SCCmec type III were divided into two groups, one of them related to the Brazilian endemic clone (HU25). According to Vivone et al. [28], this clone is responsible for most infections caused by MRSA. The MRSA isolates carrying SCCmec type IV could also be divided into two groups, one related to clone JCSC4469 and the other related to clone USA800. The strain mentioned above, which was isolated in 1993 and carried SCCmec type IV, was related to clone USA800. This group comprised strains isolated between 1993 and 2005. Trindade et al. [29] found a variety of MRSA that were related to the Brazilian endemic clone. In the present study, strains related to the Brazilian endemic clone predominated until 2003, whereas strains related to clones JCSC4469 and USA800 were found after this period. A Brazilian study conducted in a university hospital that analyzed clonal groups over a period of 8 years found that the clones identified were replaced over time, without any predominance in a specific hospital area [30]. According to the authors, replacement of clonal groups over time might be explained by microevolution of the pathogen or by competition to adapt to the hospital environment. Furthermore, the report of the presence of the pediatric clone in central Brazil suggests that this clone is settling in Brazilian hospitals and spreading in the community, increasing the likelihood of expanding its reservoir [31].

\section{Conclusions}

The clonal MRSA groups found in the NICU and PICU of HC-FMB highlight the importance of improving hygiene practices and control measures of nosocomial infections in these units since hospitalized children are generally more vulnerable because of exposure to several risk factors. Furthermore, the clonal groups that predominated over the past years carry SCCmec type IV, an element that does not impose any metabolic cost on the host and that may spread in the absence of antibiotic selective pressure. This fact may result in the emergence of this type as a new pathogen in the world. Although the sample size of this study was too small to draw any definite conclusions, according to the literature, community- 
associated MRSA are steadily increasing and may replace or be the more dominant population in clinical settings.

\section{Abbreviations}

MRSA: Methicillin-resistant Staphylococcus aureus; NICU: Neonatal intensive care units; PICU: Pediatric intensive care units; PBP 2a: Penicillin-binding protein; SCCmec: Staphylococcal cassette chromosome mec; PFGE: Pulsed-field gel electrophoresis; HC-FMB: University hospital of the Botucatu medical school.

\section{Competing interests}

The authors declare that they have no competing interests.

\section{Authors' contributions}

VCP: Conceived and designed the study, performed the microbiological tests, and wrote the article. DFMR: Participated in the PFGE analysis. MLRSC: responsible for the conception and design of the study, coordination of laboratory work, data analysis and manuscript writing. All authors read and approved the final manuscript.

\section{Acknowledgements}

Fundação de Amparo à Pesquisa do Estado de São Paulo (FAPESP; grant 2005/02830-4).

Received: 14 May 2014 Accepted: 17 October 2014

Published online: 07 November 2014

\section{References}

1. Garrit GM, Bell JA, Liburg TG: Taxonomic Outline of the Procaryotic Genera. In Bergey's Manual of Systematic Bacteriology. 2nd edition. New York: Springer Verlag; 2004.

2. Euzéby JP: List of Prokaryotic names with Standing in Nomenclature - Genus Staphylococcu; 2014. http://www.bacterio.net/staphylococcus.html.

3. Bergdoll MS: Staphylococcus aureus. J Assoc Off Anal Chem 1991, 74:706-710.

4. Bannerman TL: Staphylococcus, Micrococcus and other catalase-positive cocci that grow aerobically. In Manual of Clinical Microbiology. Edited by Baron EJ, Jorgensen JH, Pfaller MA, Yolken RH. Washington DC: American Society Microbiology; 2001:384-404.

5. Kuehnert MJ, Kruszon-Moran D, Hill HA, McQuillan G, McAllister K, Fosheim G, McDougal LK, Chaitram J, Jensen B, Fridkin SK, Killgore G, Tenover FC: Prevalence of Staphylococcus aureus nasal colonization in the United States, 2001-2002. J Infect Dis 2006, 193:172-179.

6. Kuint J, Barzilai A, Regev-Yochay G, Rubinstein E, Keller N, Maayan-Metzger A: Comparison of community-acquired methicillin-resistant Staphylococcus aureus bacteremia to other staphylococcal species in a neonatal intensive care unit. Eur J Pediatrics 2007, 166(4):319-325.

7. Archer G, Niemeyer DM: Origin and evolution of DNA associated with resistance to methicillin in Staphylococci. Trends in Microbiol 1994, 2:343-347.

8. International Working Group on the Classification of Staphylococcal Cassette Chromosome Elements (IVG-SCC): Classification of Staphylococcal Cassette Chromosome mec (SCCmec): Guidelines for Reporting Novel SCCmec Elements. Antimicrob Agents Chemother 2009, 53:4961-4967.

9. IWG-SCC: International Working Group on the Classification of Staphylococcal Cassette Chromosome Elements; 2012. http://www.sccmec.org.

10. Deresinski S: Methicillin-resistant Staphylococcus aureus: an evolutionary, epidemiologic, and therapeutic odyssey. Clin Infect Dis 2005, 40(4):562-573.

11. Ito T, Katayama Y, Hiramatsu K: Cloning and nucleotide sequence determination of the entire mec DNA of pre-methicillin-resistant Staphylococcus aureus N315. Antimicrob Agents Chemother 1999, 43:1449-1458.

12. Ito T, Katayama Y, Asada K, Mori N: Structural comparison of three types of staphylococcal cassette chromosome mec integrated in the chromosome in methicillin-resistant Staphylococcus aureus. Antimicrob Agents Chemother 2001, 45:1323-1336.

13. Sloos JH, Dijkshoorn L, Vogel L, Van Boven CPA: Performance of phenotypic and genotypic methods to determine the clinical relevance of serial blood isolates of Staphylococcus epidermidis in patients with septicemia. J Clin Microbiol 2000, 38:2488-2493.

14. Koneman EW, Allen SD, Janda WM, Schreckenberger PC, Winn WC Jr: Color Atlas and Textbook of Diagnostic Microbiology. 5th edition. Philadelphia: Lippincott; 1997:1395.

15. Murakami K, Minamide K, Wada K, Nakamura E, Teraoka H, Watanabe S: Identification of methicillin-resistant strains of staphylococci by polymerase chain reaction. J Clin Microbiol 1991, 29:2240-2244.

16. Milheiriço C, Oliveira DC, Lencastre H: Update to the multiplex PCR strategy for assignment of mec element types in Staphylococcus aureus. Antimicrob Agents Chemother 2007, 51(9):3374-3377.

17. McDougal LK, Steward CD, Killgore GE, Chaitram JM, McAllister SK, Tenover FC: Pulsed-field gel electrophoresis typing of oxacillin-resistant Staphylococcus aureus isolates from the United States: establishing a national database. J Clin Microb 2003, 41:5113-5120.

18. Denniston S, Andrew F, Riordan I: Staphylococcus aureus bacteraemia in children and neonates: a 10 year retrospective review. J Infection 2006, 53:387-393.

19. Miles F, Voss L, Segedin E, Anderson BJ: Review os Staphylococcus aureus infection requiring admission to a Paediatric Intensive Care Unit. Arch Dis Child 2005, 90:1274-1278.

20. Dolapo O, Dhanireddy R, Talati AJ: Trends of Staphylococcus aureus bloodstream infections in a neonatal intensive care unit from 2000-2009. BMC Microbiol 2014, 14:121. doi:10.1186/1471-2431-14-121.

21. Healy CM, Hulten KG, Palazzi DL, Campbell JR, Baker KJ: Emergence of new strains of methicillin-resistant Staphylococcus aureus in a neonatal intensive care unit. Clin Infect Dis 2004, 39:1460-1466.

22. Amorim ML, Faria NA, Oliveira DC, Vasconcelos C, Cabeda JC, Mendes AC, Calado E, Castro AP, Ramos MH, Amorim JM, de Lencastre H: Changes in the clonal nature and antibiotic resistance profiles of methicillin-resistant Staphylococcus aureus isolates associated with spread of the EMRSA-15 clone in a tertiary care Portuguese hospital. J Clin Microbiol 2007, 45(9):2881-2888.

23. Aires-de-Sousa M, Correia B, de Lencastre H: Changing patterns in frequency of recovery of five methicillin-resistant Staphylococcus aureus clones in Portuguese hospitals: surveil- lance over a 16-year period. J Clin Microbiol 2008, 46(9):2912-2917.

24. Daum RS, Ito T, Hiramatsu K, Hussain F, Mongkolrattanothai K, Jamklang M, Boyle-Vavra S: A novel methicillin-resistance cassette in communityacquired methicillin-resistant Staphylococcus aureus isolates of diverse genetic backgrounds. J Infect Dis 2002, 186:1344-1347.

25. Machado ABMP, Reiter KC, Paiva RM, Barth AL: Distribution of staphylococcal cassette chromosome mec (SCCmec) types I, II, III and IV in coagulasenegative staphylococci from patients attending a tertiary hospital in southern Brazil. J Med Microbio/ 2007, 56:1328-1333.

26. Martins A, Riboli DFM, Pereira VC, Cunha MLRS: Molecular characterization of methicillin-resistant Staphylococcus aureus isolated from a Brazilian university hospital. Braz J Infect Dis 2014, 18(3):331-335.

27. Perez $L R, D^{\prime}$ Azevedo PA: Clonal types and antimicrobial resistance profiles of methicillin-resistance Staphylococcus aureus isolates from hospitals in south Brazil. Rev Inst Med Trop Sao Paulo 2008, 50(3):135-137.

28. Vivone AM, Diep BA, Gouveia Magalhães AC, Santos KR, Riley LW, Sensabaugh GF, Moreira BM: Clonal composition of Staphylococcus aureus isolates at a Brazilian university hospital: identification of international circulating lineages. J Clin Microbiol 2006, 44(5):1686-1691.

29. Trindade PA, Pacheco RL, Costa SF, Rossi F, Barone AA, Mamizuka EM, Levin AS: Prevalence of SCCmec type IV in nosocomial bloodstream isolates of methicillin-resistant Staphylococcus aureus. J Clin Microbiol 2005, 43(7):3435-3437.

30. Leite GC, Padoveze MC, Moretti ML: Methicillin-resistant Staphylococcus aureus DNA electrophoretic pattern: temporal changes in an endemic hospital environment. Rev Panam Salud Publica 2011, 30(6):535-539.

31. Vieira MA, Minamisavab R, Pessoa-Júnior V, Lamaro-Cardoso J, Ternesc YM, Andre MCP, Sgambatti S, Kipnis A, Andrade AN: Methicillin-resistant Staphylococcus aureus nasal carriage in neonates and children attending a pediatric outpatient clinics in Brazil. Braz J Infect Dis 2014, 18(1):42-47.

doi:10.1186/s12941-014-0050-4

Cite this article as: Pereira et al:: Characterization of the clonal profile of MRSA isolated in neonatal and pediatric intensive care units of a University Hospital. Annals of Clinical Microbiology and Antimicrobials 2014 13:50. 